\title{
Qualitative properties and hopf bifurcation in haematopoietic disease model with chemotherapy
}

\author{
R. Yafia ${ }^{1, a}$, M. A. Aziz-Alaoui ${ }^{2, b}$, A. Tridane ${ }^{3, c}$, and F. Rihan ${ }^{4, d}$ \\ 1 Ibnou Zohr University, Polydisciplinary Faculty of Ouarzazate, B.P: 638, Ouarzazate, Morocco. \\ 2 Laboratoire de Mathématiques Appliquées, 25 Rue Ph. Lebon, BP 540, 76058Le Havre Cedex, France. \\ 3 Department of Mathematical Sciences College of Science, UAE University P.O. Box 17551, Al-Ain United Arab Emi- \\ rates. \\ 4 Department of Mathematical Sciences College of Science, UAE University P.O. Box 17551, Al-Ain United Arab Emi- \\ rates.
}

\begin{abstract}
In this paper, we consider a model describing the dynamics of Hematopoietic Stem Cells (HSC) disease with chemotherapy. The model is given by a system of three ordinary differential equations with discrete delay. Its dynamics are studied in term of local stability of the possible steady states for the case without drug intervention term. We prove the existence of periodic oscillations for each case when the delay passes trough a critical values. In the end, we illustrate our results by some numerical simulations.
\end{abstract}

\section{Introduction}

In this paper, we propose a model of Haematopoietic stem cells which in we incorporate the chemotherapy $[1,8]$. The variation of chemotherapy is described by the following equation:

$$
\frac{d \gamma_{c}(t)}{d t}=m(t)-k \gamma_{c}(t)
$$

where $m(t)$ is a function of time which represents the drug intervention term and $k$ is the per capita decay rate and $\gamma_{c}(t)$ the biomass of chemotherapy at time $t$.

The full model is given by:

$$
\left\{\begin{array}{c}
\frac{d N}{d t}=-\delta N-\beta(N) N+2 e^{-\gamma \tau-\int_{t-\tau}^{t} \gamma_{c}(s) d s} \beta\left(N_{\tau}\right) N_{\tau} \\
\frac{d P}{d t}=-\gamma_{1}(t) P+\beta(N) N-e^{-\gamma \tau-\int_{t-\tau}^{t} \gamma_{c}(s) d s} \beta\left(N_{\tau}\right) N_{\tau} \\
\frac{d \gamma_{c}(t)}{d t}=m(t)-k \gamma_{c}(t)
\end{array}\right.
$$

where $\beta(N)=\beta_{0} \frac{\theta^{n}}{\theta^{n}+N^{n}}$ and $N$ is the number of cells in nonproliferative phase, $N_{\tau}=N(t-\tau), P$ the number of cycling proliferating cells, $\gamma$ the rate of cells loss from proliferative phase, $\delta$ the rate of cells loss from non-proliferative phase, $\tau$ the time spent in the proliferative phase, $\beta$ the feedback function, rate of recruitment from non-proliferative phase, $\beta_{0}>0$ the maximal rate of re-entry in the proliferating phase, $\theta \geq 0$ is the number of resting cells at which $\beta$ has its maximum rate of change with respect to the resting phase population, $n>0$ describes the sensitivity of reintroduction rate with changes in the population, and $e^{-\gamma \tau}$ accounts for the attenuation due to apoptosis (programmed cell death) at rate $\gamma$.

Normalizing the delay $\tau$ by the time scaling $t \rightarrow \frac{t}{\tau}$, effecting the change of variables $u(t)=N(t \tau)$ and $v(t)=P(t \tau)$

\footnotetext{
a e-mail: yafia1@yahoo.fr

b e-mail: aziz.alaoui@univ-lehavre.fr

c e-mail: a-tridane@uaeu.ac.ae

d e-mail: frihan@uaeu.ac.ae
}

and $w(t)=\gamma_{c}(t \tau)$ the system (2) is transformed into

$$
\begin{gathered}
\dot{u}(t)=\tau\left[-\delta u(t)-\alpha(u(t))+2 e^{-\gamma \tau-\tau \int_{t-1}^{t} w(s) d s} \alpha(u(t-1))\right] \\
\left\{\dot{v}(t)=\tau\left[(-\gamma-w(t)) v(t)+\alpha(u(t))-e^{-\gamma \tau-\tau \int_{t-1}^{t} w(s) d s} \alpha(u(t-1))\right]\right. \\
\dot{w}(t)=\tau(m(t)-k w(t))
\end{gathered}
$$

where $\alpha(x)=\beta(x) x$

The current work is organized as follows: In section 2 , we study the model in the absence of the drug intervention term $(m(t)=0)$, we prove the asymptotic stability of the possible steady states and the existence of periodic oscillations. We end our paper by section 3 in which we give a numerical study of our results.

\section{Model with $m(t)=0$}

In this section, we consider model (3) without the drug intervention term that is $m(t)=0$ and the model becomes:

$$
\begin{gathered}
\dot{u}(t)=\tau\left[-\delta u(t)-\alpha(u(t))+2 e^{-\gamma \tau-\tau \int_{t-1}^{t} w(s) d s} \alpha(u(t-1))\right] \\
\left\{\dot{v}(t)=\tau\left[(-\gamma-w(t)) v(t)+\alpha(u(t))-e^{-\gamma \tau-\tau \int_{t-1}^{t} w(s) d s} \alpha(u(t-1))\right]\right. \\
\dot{w}(t)=-\tau k w(t)
\end{gathered}
$$

\subsection{Steady states and stability}

The steady states of the model (4) are given by the resolution of the following equation

$$
\frac{d u}{d t}=\frac{d v}{d t}=\frac{d w(t)}{d t}=0
$$

From the equation $(4)_{3}$, we obtain that $w=0$ then we solve the following equations

$$
\left\{\begin{array}{c}
-\delta u(t)-\alpha(u(t))+2 e^{-\gamma \tau} \alpha(u(t-1))=0 \\
-\gamma v(t)+\alpha(u(t))-e^{-\gamma \tau} \alpha(u(t-1))=0
\end{array}\right.
$$


Let

$\left(\mathbf{H}_{0}\right) \delta<\frac{\beta_{0}}{2}$.

Denote by $\bar{\tau}=\frac{1}{\gamma} \ln \left(\frac{2}{1+\frac{2 \delta}{\beta_{0}}}\right)$.

Note that $\left(\mathrm{H}_{0}\right)$ implies that for each $0<\tau<\bar{\tau}, \alpha^{\prime}\left(u^{*}\right)<0$ and $\beta_{0}\left(2 e^{-\gamma \tau}-1\right)>\delta$ and system (4) has a unique positive equilibrium $E^{*}(\tau)=\left(u^{*}(\tau), v^{*}(\tau), 0\right)$ with

$u^{*}(\tau)=\theta\left(\frac{\beta_{0}\left(2 e^{-\gamma \tau}-1\right)-\delta}{\delta}\right)^{1 / n}, \quad v^{*}(\tau)=\frac{\delta u^{*}}{\gamma}\left(\frac{1-e^{-\gamma \tau}}{2 e^{-\gamma \tau}-1}\right)$

The following result gives the existence of possible steady states of system (4)

Proposition 1 If $\left.\left(H_{0}\right)\right)$ is not satisfied, the system (4) has a unique steady state which is trivial $E_{0}=(0,0,0)$. If $\left.\left(H_{0}\right)\right)$ is satisfied, the system (4) has two equilibrium points, the first is trivial $E_{0}=(0,0,0)$ and the second is nontrivial and positive which given by $E^{*}(\tau)=\left(u^{*}(\tau), v^{*}(\tau), 0\right)$.

For the stability, we begin by studying the stability of the trivial steady state $E_{0}=(0,0,0)$. By linearizing the system (4) around $E_{0}$, we have the following linearized equation:

$$
\left\{\begin{array}{c}
\dot{u}(t)=\tau\left[-\delta u(t)-\beta_{0} u(t)+2 e^{-\gamma \tau} \beta_{0} u(t-1)\right] \\
\dot{v}(t)=\tau\left[-\gamma v(t)+\beta_{0} u(t)-e^{-\gamma \tau} \beta_{0} u(t-1)\right]
\end{array}\right.
$$

The associated characteristic equation is as follows:

$$
(\lambda+\tau k)(\lambda+\tau \gamma)\left(\lambda+\tau \delta+\tau \beta_{0}-2 \tau e^{-\gamma \tau} \beta_{0} e^{-\lambda}\right)=0
$$

As $\lambda_{1}=-\tau k<0$ and $\lambda_{2}=-\tau \gamma<0$ one need to study the following equation:

$$
\lambda+\tau \delta+\tau \beta_{0}-2 \tau e^{-\gamma \tau} \beta_{0} e^{-\lambda}=0
$$

Then $E_{0}$ is asymptotically stable if and only if

$$
2 \beta_{0} e^{-\gamma \tau}<\delta+\beta_{0}
$$

To study the stability of $E^{*}(\tau)=\left(u^{*}(\tau), v^{*}(\tau), 0\right)$ one needs to linearize system $(4)$ around $E^{*}(\tau)$.

By the translation $z(t)=(u(t), v(t), w(t))-\left(u^{*}(\tau), v^{*}(\tau), 0\right)$, system (4) is written as a functional differential equation $(\mathrm{FDE})$ in $C:=C\left([-1,0], \mathbb{R}^{3}\right)$ :

$$
\dot{z}(t)=L(\tau) z_{t}+f_{0}\left(z_{t}, \tau\right)
$$

where $L(\tau): C \longrightarrow \mathbb{R}^{3}$ is a linear operator and $f_{0}: C \times$ $\mathbb{R} \longrightarrow \mathbb{R}^{3}$ are given respectively by:

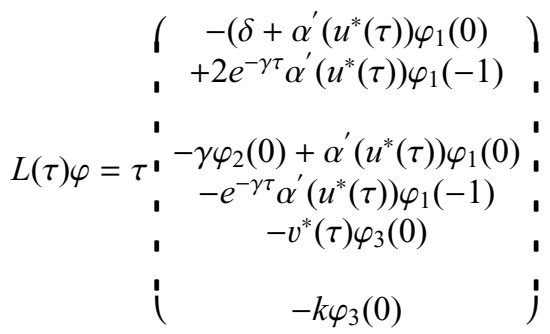

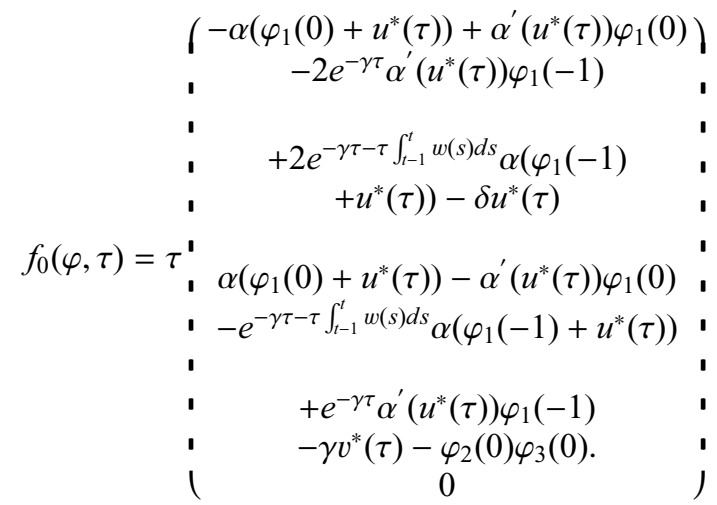

for $\varphi=\left(\varphi_{1}, \varphi_{2}, \varphi_{3}\right) \in C$.

The characteristic equation of the linear equation $\dot{z}(t)=$ $L(\tau) z_{t}$ is given by

$$
W(\lambda, \tau)=(\lambda+\tau k)(\lambda+\tau \gamma)\left(\lambda-\tau a(\tau)-\tau b(\tau) e^{-\lambda}\right)=0,
$$

with $a(\tau)=-\left(\delta+\alpha^{\prime}\left(u^{*}(\tau)\right)\right)$ and $b(\tau)=2 e^{-\gamma \tau} \alpha^{\prime}\left(u^{*}(\tau)\right)$ and $\alpha^{\prime}\left(u^{*}(\tau)\right)=\frac{\delta}{\beta_{0}\left(2 e^{-\gamma \tau}-1\right)^{2}}\left[\beta_{0}(1-n)\left(2 e^{-\gamma \tau}-1\right)+n \delta\right]$.

As $\tau \gamma>0$ and $\tau k>0$, the stability of the equilibrium $z=0$ follows from the study of the roots of the following equation:

$$
\Delta(\lambda, \tau)=\lambda-\tau a(\tau)-\tau b(\tau) e^{-\lambda}=0
$$

corresponding to the characteristic equation associated to the first equation in (10). To obtain the switch of stability of $z=0$ one needs to find the imaginary root of equation (12). Let $\lambda=i \zeta$, then $\Delta(i \zeta, \tau)=0$ if and only if

$$
\left\{\begin{array}{l}
\zeta=\arccos \left(-\frac{a(\tau)}{b(\tau)}\right) \in(0, \pi) \text { for } 0 \leq\left.{ }^{\mid} \frac{a(\tau)}{b(\tau)}\right|_{\mid} \leq 1 \\
\quad \text { and } \\
\left\{\sqrt{b^{2}(\tau)-a^{2}(\tau)}=\arccos \left(-\frac{a(\tau)}{b(\tau)}\right) \text { for } 0 \leq{ }_{\mid}^{\mid} \frac{a(\tau)}{b(\tau)} \mid<1 .\right.
\end{array}\right.
$$

Let:

$\left(\mathbf{H}_{1}\right): \quad a(\tau)<0$ and $|b(\tau)|<-a(\tau)$ for all $\tau>0$.

$\left(\mathbf{H}_{2}\right): \quad \tau a(\tau)<1$, and $|a(\tau)|<|b(\tau)|$ for all $\tau>0$.

Theorem 1 Assume $\left(\mathbf{H}_{\mathbf{0}}\right)$. Then,

(1) The trivial equilibrium $E_{0}=(0,0,0)$ of system (4) is unstable for $0<\tau<\bar{\tau}$.

(2) (i) If $a$ and b satisfy $\left(\mathbf{H}_{\mathbf{1}}\right)$, then the equilibrium point $z=0$ of (10) is asymptotically stable for $0<\tau<\bar{\tau}$.

(ii) If $a$ and $b$ satisfy $\left(\mathbf{H}_{2}\right), n \geq 2$ and $\gamma$ close enough to 0 , there exists a unique $\tau_{0}$ in $] 0, \bar{\tau}$ [ such that the equilibrium point $z=0$ of (10) is asymptotically stable for $\tau \in] 0, \tau_{0}[$ and unstable for $\tau \in\left(\tau_{0}, \bar{\tau}\right)$.

Proof (1) The characteristic equation of the linearized equation associated to $(4)$ around $(0,0)$ is given by,

$$
\lambda+\tau\left(\delta+\beta_{0}\right)-2 \tau e^{-\gamma \tau} \beta_{0} e^{-\lambda}=0
$$

From $\left(\mathbf{H}_{\mathbf{0}}\right)$, we have $\beta_{0}\left(2 e^{-\gamma \tau}-1\right)>\delta$, thus equation (14) has a real root which is positive. Then $E_{0}$ is unstable.

(2) (i) Let $\lambda=\mu+i v$ be a root of equation $\Delta(\lambda, \tau)=0$ for $0<\tau<\bar{\tau}$. We have:

$$
\left\{\begin{array}{c}
\mu-\tau a(\tau)-\tau b(\tau) e^{-\mu} \cos (v)=0 \\
v+\tau b(\tau) e^{-\mu} \sin (v)=0
\end{array}\right.
$$

If there exists a root $\mu_{0} \geq 0$ of (12), then $-a(\tau) \leq b(\tau) e^{-\mu_{0}} \cos (v)$. As $-1 \leq \cos (v) \leq 1$ and $0<e^{-\mu_{0}}<1$ and $b(\tau)<0$ for 
$0<\tau<\bar{\tau}$, we have $b(\tau) \leq a(\tau)$, which contradicts the assumption $\left(\mathbf{H}_{1}\right)$. So for all $0<\tau<\bar{\tau}$, the roots of the equation (12) have negative real parts, and therefore $z=0$ is asymptotically stable.

For the proof of the stability in (2) (ii), we need the following lemmas.

Lemma 1 (Hale 1993 [4]) All roots of the equation $(z+$ $c) e^{z}+d=0$, where $c$ and $d$ are real, have negative real parts if and only if,

(i) $c>-1$

(ii) $c+d>0$

(iii) $\sqrt{d^{2}-c^{2}}<\zeta$

where $\zeta$ is the root of $\zeta=-c \tan \zeta, 0<\zeta<\pi$, if $c \neq 0$ and $\zeta=\frac{\pi}{2}$ if $c=0$.

Lemma 2 Under hypotheses $\left(\mathbf{H}_{\mathbf{0}}\right)$ and $\left(\mathbf{H}_{\mathbf{2}}\right)$, for $n \geq 2$ and $\gamma$ close enough to 0 , there exists a unique solution $\tau_{0}$ of the second equation of (13) in $] 0, \bar{\tau}\left[\right.$, such that $i \zeta_{0}$ is a purely imaginary root of equation (12), with $\zeta_{0}=\arccos \left(-\frac{a\left(\tau_{0}\right)}{b\left(\tau_{0}\right)}\right)$.

Furthermore, the following inequalities hold:

$$
\left\{\begin{array}{l}
\tau \sqrt{b^{2}(\tau)-a^{2}(\tau)}<\arccos \left(-\frac{a(\tau)}{b(\tau)}\right) \text { for } \tau \in\left(0, \tau_{0}\right) \\
\tau \sqrt{b^{2}(\tau)-a^{2}(\tau)}>\arccos \left(-\frac{a(\tau)}{b(\tau)}\right) \text { for } \tau \in\left(\tau_{0}, \bar{\tau}\right)
\end{array}\right.
$$

Lemma 3 Let $f:(0, \pi) \rightarrow \mathbb{R}$ be defined by $f(x)=\alpha \tan x$, $\alpha<1$ and $\alpha \neq 0$. Then, $f$ has a unique fixed point $\zeta \in$ $(0, \pi)$, such that:

for $0<\alpha<1, f(x)<x$ if $x \in(0, \zeta) \cup\left(\frac{\pi}{2}, \pi\right)$ and $f(x)>x$ if $x \in\left(\zeta, \frac{\pi}{2}\right)$,

and for $\alpha<0, f(x)<x$ if $x \in\left(0, \frac{\pi}{2}\right) \cup(\zeta, \pi)$ and $f(x)>x$ if $x \in\left(\frac{\pi}{2}, \zeta\right)$.

Proof of (2)(ii) of theorem 1.

We only have to verify the three conditions (i), (ii) and (iii) of lemma 1. The assertions (i) and (ii) follow from $\left(\mathbf{H}_{2}\right)$ with $c=-\tau a(\tau)$ and $d=-\tau b(\tau)$.

For (iii), let $\tau \in\left(0, \tau_{0}\right)$ and $f(\zeta)=\tau a(\tau) \tan \zeta$. From the first equation of (16):

if $a(\tau)=0$, the first inequality of $(16)$ becomes $-\tau b(\tau)<\frac{\pi}{2}$, and (iii) is satisfied.

If $0<\tau a(\tau)<1$ or $a(\tau)<0$, as

$$
f\left(\arccos \left(-\frac{a(\tau)}{b(\tau)}\right)\right)=\tau \sqrt{b(\tau)^{2}-a(\tau)^{2}},
$$

the first equation of (16) implies that

$$
f\left(\arccos \left(-\frac{a(\tau)}{b(\tau)}\right)\right)<\arccos \left(-\frac{a(\tau)}{b(\tau)}\right),
$$

with $\arccos \left(-\frac{a(\tau)}{b(\tau)}\right) \in(0, \pi)$.

From lemma 3 and the graph of $f$, if $\zeta$ is the fixed point of $f$ in $(0, \pi)$, we have,

$$
f\left(\arccos \left(-\frac{a(\tau)}{b(\tau)}\right)\right)<\zeta
$$

that is

$$
\sqrt{(\tau b(\tau))^{2}-(\tau a(\tau))^{2}}<\zeta,
$$

which leads to the desired assertion. This complete the stability of $z=0$ for $0<\tau<\tau_{0}$.

To prove the instability of $z=0$ in (2) (ii), for $\tau_{0}<\tau<\bar{\tau}$, we will show that the characteristic equation (12) has at least one root with positive real part.

Let $\tau_{0}<\tau<\bar{\tau}$. If all the roots of the characteristic equation (12) have negative real parts, the properties (i), (ii) and (iii) of lemma 1 are satisfied. From the second equation of (16) and from (17) we have,

$$
\left\{\begin{array}{c}
f\left(\arccos \left(-\frac{a(\tau)}{b(\tau)}\right)\right)>\arccos \left(-\frac{a(\tau)}{b(\tau)}\right) \\
\text { and } \\
f\left(\arccos \left(-\frac{a(\tau)}{b(\tau)}\right)\right)<\bar{\zeta}
\end{array}\right.
$$

Henceforth, from lemma 3 and the graph of $f$, we have

$$
\left\{\begin{array}{r}
\arccos \left(-\frac{a(\tau)}{b(\tau)}\right)<\bar{\zeta} \\
\text { and } \\
\arccos \left(-\frac{a(\tau)}{b(\tau)}\right)>\bar{\zeta}
\end{array}\right.
$$

which is impossible.

Now, suppose that there is one root with zero real part with all the remaining roots having negative real parts. From (13) and lemma 2 we deduce that $\tau=\tau_{0}$, which contradicts the assumption $\tau>\tau_{0}$. Then $z=0$ is unstable for $\tau_{0}<\tau<$ $\bar{\tau}$.

Proof (of lemma 2)

In view of $\left(\mathbf{H}_{\mathbf{0}}\right)$ and $\left(\mathbf{H}_{\mathbf{2}}\right)$, to find a root of second equation of (13) is equivalent to find a root of the equation

$$
\tau=-\frac{\arccos \left(-\frac{a(\tau)}{b(\tau)}\right)}{b(\tau) \sin \left(\arccos \left(-\frac{a(\tau)}{b(\tau)}\right)\right)} .
$$

Let: $y(\tau)=\arccos \left(-\frac{a(\tau)}{b(\tau)}\right)$, and $F(\tau)=-\frac{y(\tau)}{b(\tau) \sin (y(\tau))}$.

Besides, in the hypotheses $\left(\mathbf{H}_{\mathbf{0}}\right)$ and $\left(\mathbf{H}_{\mathbf{2}}\right), F$ is continuously differentiable on $\tau \in[0, \bar{\tau}]$. As $y(0) \in(0, \pi)$ and $b(0)<0$, we have $F(0)>0$ for $n \geq 2$ and $F(\bar{\tau})<\bar{\tau}$ for $\gamma$ close enough to 0 (because $F(\bar{\tau})$ is independent of $\gamma$ and $\bar{\tau}$ is larger when $\gamma$ is small), then there exits at least one solution $\tau_{0}$ of equation (18) in ]0, $\bar{\tau}[$. Now, for the uniqueness of $\tau_{0}$, let $g(\tau)=\tau-F(\tau)$, then

$$
\begin{gathered}
g^{\prime}(\tau)=1-\frac{y^{\prime}(\tau) b(\tau) \sin (y(\tau))-y(\tau) b^{\prime}(\tau) \sin (y(\tau))}{(b(\tau) \sin (y(\tau)))^{2}} \\
-\frac{y(\tau) b(\tau) \cos ((\tau)) y^{\prime}(\tau)}{(b(\tau) \sin (y(\tau)))^{2}}
\end{gathered}
$$

where

$$
y^{\prime}(\tau)=-\sqrt{1-\left(\frac{a(\tau)}{b(\tau)}\right)^{2}} \frac{a^{\prime}(\tau) b(\tau)-a(\tau) b^{\prime}(\tau)}{b^{2}(\tau)} .
$$

As

$$
\lim _{\gamma \rightarrow 0} \frac{d}{d \tau} \alpha^{\prime}\left(u^{*}\right)=0
$$

from (11), we have:

$$
\lim _{\gamma \rightarrow 0} b^{\prime}(\tau)=0 \quad \text { and } \quad \lim _{\gamma \rightarrow 0} a^{\prime}(\tau)=0 .
$$

Then

$$
\lim _{\gamma \rightarrow 0} g^{\prime}(\tau)=1>0, \text { for all } 0 \leq \tau \leq \bar{\tau}
$$

so as, $g^{\prime}>0$ and $g$ is an increasing function on the interval ] $0, \bar{\tau}$ [ for $\gamma$ close enough to $0, \tau_{0}$ is unique in $] 0, \bar{\tau}[$. By the continuity property of $F$, we have $F(\tau)>\tau$ for $\tau \in] 0, \tau_{0}[$ and $F(\tau)<\tau$ for $\tau \in] \tau_{0}, \bar{\tau}[$.

The proof of lemma 3 is obvious. 


\subsection{Hopf bifurcation}

From theorem 1, we deduce that the Hopf bifurcation may occurs at the critical value $\tau=\tau_{0}$ and we have the following result.

Theorem 2 Assume $\left(\mathbf{H}_{\mathbf{0}}\right),\left(\mathbf{H}_{\mathbf{2}}\right), n \geq 2$ and $\gamma$ sufficiently small. The system (10) has a Hopf bifurcation as the delay crosses the critical value $\tau=\tau_{0}$ which is stated in lemma 2.

Proof We apply the Hopf bifurcation theorem (see [4]). From the expression of $f_{0}$ in (??), we have,

$$
f_{0}(0, \tau)=0 \quad \text { and } \quad \frac{\partial f_{0}(0, \tau)}{\partial \varphi}=0, \text { for all } \tau>0
$$

From (12) and lemma 2, we have:

$$
\Delta(i \zeta, \tau)=0 \quad \Leftrightarrow \quad\left\{\begin{array}{c}
\zeta=\zeta_{0}=\arccos \left(-\frac{a\left(\tau_{0}\right)}{b\left(\tau_{0}\right)}\right) \\
\text { and } \\
\tau=\tau_{0}
\end{array}\right.
$$

Thus, characteristic equation (11) has a pair of simple imaginary roots $\lambda_{0}=i \zeta_{0}$ and $\bar{\lambda}_{0}=-i \zeta_{0}$ at $\tau=\tau_{0}$.

Lastly, we need to verify the transversality condition.

From $(11), W\left(\lambda_{0}, \tau_{0}\right)=0$ and $\frac{\partial}{\partial \lambda} W\left(\lambda_{0}, \tau_{0}\right)=\left(\lambda_{0}+\gamma \tau_{0}\right)(1-$ $\left.\tau_{0} a\left(\tau_{0}\right)+\lambda_{0}\right) \neq 0$. According to the implicit function theorem, there exists a complex function $\lambda=\lambda(\tau)$ defined in a neighborhood of $\tau_{0}$, such that $\lambda\left(\tau_{0}\right)=\lambda_{0}$ and $\Delta_{0}(\lambda(\tau), \tau)=$ 0 and

$$
\lambda^{\prime}(\tau)=-\frac{\partial W(\lambda, \tau) / \partial \tau}{\partial W(\lambda, \tau) / \partial \lambda}, \text { for } \tau \text { in a neighborhood of } \tau_{0} .
$$

Let $\lambda(\tau)=p(\tau)+i q(\tau)$. From (19) we have:

$$
p^{\prime}(\tau)_{/ \tau=\tau_{0}}=\frac{1}{\tau_{0}} \frac{\zeta_{0}^{2}}{\left(1-\tau_{0} a\left(\tau_{0}\right)\right)^{2}+\zeta_{0}^{2}} \text { for } \gamma=0
$$

By the continuity property, we conclude that, $p^{\prime}(\tau)_{/ \tau=\tau_{0}}>$ 0 , for $\gamma$ close to 0 .

\section{Numerical simulations}

With Matlab software, we give numerical simulations of this theoretical results. We compare the size of stem cells in each phase of cycle (resting and proliferating phases), we prove that the chemotherapy can reduce the size of each type of cells.

In table 1 we give all used parameters.

\section{References}

1. L. K. Andersen and M. C. Mackey, Resonance in Periodic Chemotherapy: A Case Study of Acute Myelogenous Leukemia. J. theor. Biol. 209 (2001) 113-130.

2. S. Chareyron andM. Alamir,Mixed immunotherapy and chemotherapy of tumors: Feedback design and model updating schemes, Journal of Theoretical Biology,(2008) doi:10.1016/j.jtbi.2008.07.002, .
Table 1. Parameters estimation and their references:.

\begin{tabular}{cccc}
\hline Parameters & Value used & Unit & Sources \\
\hline$\beta_{0}$ & $3-3.5$, & day $^{-1}$ & {$[6,3]$} \\
$\theta$ & $1.38 \times 10^{8}-0.5 \times 10^{6}$ & cells kg $^{-1}$ & {$[6,3]$} \\
$n$ & $3-4$ & & {$[6,3]$} \\
$\delta$ & 0.16 & day $^{-1}$ & {$[6]$} \\
$\gamma$ & $0.1-0.36$ & day $^{-1}$ & {$[6,3]$} \\
$m$ & $<m_{\max }=1$ & $\mathrm{mg}^{\mathrm{day}}$ & {$[2]$} \\
$\tau$ & $0.83-0.88$ & $\mathrm{day}^{-1}$ & {$[6,3]$} \\
$k$ & $0.01 \times 10^{-2}-1 \times 10^{-2}$ & $\mathrm{mg}^{-1}$ & {$[5]$} \\
$N_{0}$ & $3 \times 10^{9}-1 \times 10^{11}$ & cells $^{-1}$ & {$[7]$} \\
$P_{0}$ & $0.6-2.6$ & gram & {$[1]$} \\
\hline
\end{tabular}

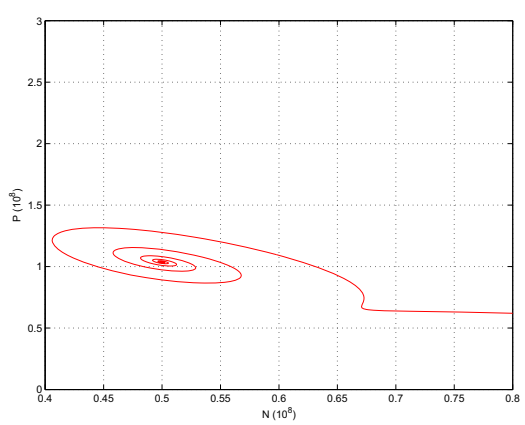

Fig. 1. Stability of the nontrivial steady state for $m=0$ and $\tau<\tau_{0}$

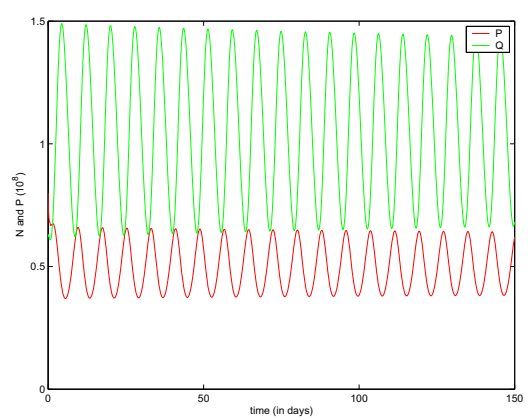

Fig. 2. Periodic oscillations around of the nontrivial steady state for $m=0$ and $\tau=\tau_{0}$

3. C. Colijn, M.C. Mackey. "A mathematical model of hematopoiesis: Periodic chronic myelogenous leukemia, part I, J. Theor. Biol. , 237, (2005) 117-132 .

4. J.K.Hale and S. M. Verduyn Lunel, Introduction to functional Differential equations. Springer-Verlag, New-York (1993).

5. W. Liu, T. Hillen, H. I. Freedman, A Mathematical model for M-phase specific chemotherapy including the $G_{0}$-phase and immunoresponse, 4, N. 2 (2007) 239-259.

6. M. C. Mackey, Cell Kenitec Status of Haematopoietic Stem Cells. cell prolif. 34 (2001) 71-83.

7. L.G. de Pillis, W.Gua, A.E. Radunskaya, Mixed immunotherapy and chemotherapy of tumors: modeling, applications and biological interpretations, Journal of Theoretical Biology 238, (2006) 841-862.

8. R. Yafia and M. A. Aziz Alaoui, Periodic Chemotherapy in Non-Specific Phase of Hematopoietic Stem Cells Model With One Delay, Advances in Dynamical Systems and 